\title{
Classifying GPR Images Using Convolutional Neural Networks
}

\author{
Maha Almaimani ${ }^{1}$, Dalei Wu ${ }^{1}$, Yu Liang ${ }^{1}$, Li Yang $^{1}$, Dryver Huston ${ }^{2}$, Tian Xia ${ }^{2}$ \\ \{dalei-wu@utc.edu, yu-liang@utc.edu, li-yang@utce.edu, dryver-huston@uvm.edu, txia@uvm.edu $\}$ \\ ${ }^{1}$ Department of Computer Science and Engineering \\ University of Tennessee at Chattanooga, Chattanooga, TN USA \\ ${ }^{2}$ School of Engineering \\ University of Vermont, Burlington, VT USA
}

\begin{abstract}
This paper focused on classifying ground penetrating radar (GPR) images of subsurface cylinders according to their depth, size, material, and the dielectric constant of the underlying medium using four different architectures of convolutional neural networks. Two CNNs were newly proposed in this study and then compared to two others that were used by other authors. These CNNs were trained by using a couple of adjusted training options including initial learning rate, learning rate drop factor, and learning rate drop period; which had a positive impact on some of the considered models, while the option maximum number of epochs worked well with all of the considered models. Results showed that the first proposed CNN showed superior performance due to the use of a deep network with a large number of small filters. It was also found that the first proposed CNN could obtain the best results when GPR B-scans were classified according to the cylinders' materials.
\end{abstract}

Keywords: GPR, Convolutional neural networks, image analysis, underground

\section{Introduction}

Ground penetrating radar (GPR) is a very common, non-destructive, subsurface imaging tool used in many applications related to infrastructure evaluations such as: landmine detection [1], pipeline detection [2], avalanche victims detection [3] [4], liquid contamination detection [5], soil contamination measurement [6], bridge deck inspection [7], etc. These applications have mainly focused on detecting and classifying buried objects according to their characteristics. These characteristics include: object depth, size, material, dielectric constant of the underground medium, etc. Currently, a few research projects focus on classifying GPR images according to their characteristics. Due to the importance of GPR use in infrastructure applications, an automatic methodology for interpreting GPR images is very necessary.

Many techniques have been employed to classify GPR images. One technique is based on using Support Vector Machines (SVMs). SVMs were used to identify the materials of underground utilities [8]. The research in [8] suggested that the segment length of A-scan should be adjusted in order to obtain more accurate results using SVMs. Furthermore, B-scan information such as feature of amplitude and frequency should be added to the raw data. Moreover, a proper kernel function and a convenient range for data normalization should be selected to obtain accurate classification results. Neural networks were also used to classify GPR images. The work in [9] exploited three neural network algorithms to simultaneously estimate the shape, material type, size and depth of the buried objects as well as the dielectric 
constant of the underground medium. The results showed that an error emerged when a circular metal object was recognized. Furthermore, triangle size estimation demonstrated that improvement in the proposed methodology was needed to achieve more accurate results. Neural networks also show limited performance with nonlinear, high dimensional samples. Thus, they can be really slow in data training and have challenges in dealing with complicated real world applications. They are also sensitive to learning samples and they have limited generalization ability [10]. Neural networks suffer from several limitations; therefore, deep convolutional neural networks were introduced to produce more accurate results.

Convolutional neural networks (CNNs) have revolutionized the field of computer vision and image detection since 2012. CNNs are deep learning neural networks where each neuron accepts inputs from neurons on the previous layer, with no existence of cycles; thus, they are called feed-forward CNNs [11]. They differ from shallow neural networks that consist of only one layer; therefore, by using filters they do not struggle with computational complexity when the input size is dramatically increased. Furthermore, in contrast to other deep neural networks, CNNs directly work on 2D images. They also use the Back Propagation algorithm to optimize the accuracy of predicting models by reducing the error related to each neuron. CNNs have been used in the ground penetrating radar field in some work [1], [12], [13] to detect buried targets. Until recently, CNNs were used only to detect objects, without classifying GPR images according to their characteristics (i.e. depth, size, material, etc.). This research investigates CNNs for GPR image classification.

The major research objective of this work is to determine the effect of different training options on four CNNs. Two of these networks were newly proposed and the other two were used by [1] and [14]. Other objectives are to determine the best combination of training options for each of the suggested CNNs, to identify which of these four models would work best in classifying GPR images according to their characteristics: depth, material, size, and the dielectric constant of the underground medium, and finally to examine which of the four characteristics results in the best classification performance.

The rest of this paper is organized as follows: Section 2 describes the methodology of the work; Section 3 present evaluation results. Section 4 concludes the paper.

\section{Methodology}

Four software packages were used in this project: gprMax v3.1.1 to generate B-scan images; Paraview v4.3 to visualize geometry created by gprMax; HDFView v3.0.0 to preprocess the images by rescaling them; and Matlab R2017b to resize the images, convert them to gray, and feed them to the proposed CNNs for training and testing.

\subsection{Data Set Configuration}

The images used in this experiment were configured using gprMax simulation. In this simulation, GPR Ricker waveform was set using a centre frequency of $1.5 \mathrm{GHz}$, to obtain a good resolution with a reasonable penetration depth. The time window was set to 3 nanoseconds to give enough time for the waves to propagate from the transmitter and reflect back to the receiver resulting images with enough details. GPR A-scan traces were collected in a horizontal direction from left to right using 60 steps on different sized domains. The amplitude of the GPR antenna above the ground was $1 \mathrm{~mm}$.

It was assumed that different cylinders were buried in a half-space with different scenarios:

- Object material: metal, concrete, polyvinyl chloride (i.e. PVC).

- Object depth: $2 \mathrm{~cm}, 100 \mathrm{~cm}$.

- Dielectric constant of the subsurface medium: 4, 6, 8.

- Object size/radius: $20 \mathrm{~mm}, 50 \mathrm{~mm}, 100 \mathrm{~mm}, 150 \mathrm{~mm}, 200 \mathrm{~mm}$. 


\subsection{Data pre-processing}

Since the images created by gprMax had different scales (as illustrated in Figure 1), they need to be normalized to have the same scale. This was accomplished by using HDFView. GprMax produces B-scan pictures and .out files along with them. Rescaling the .out files to the smallest scale would have resulted in some loss of information. Therefore, the .out files were rescaled to the largest scale found in the images dataset, which ranges from -1451.39 to 1710.09. Figure 1 gives an example of a GPR image before and after rescaling using HDFView.

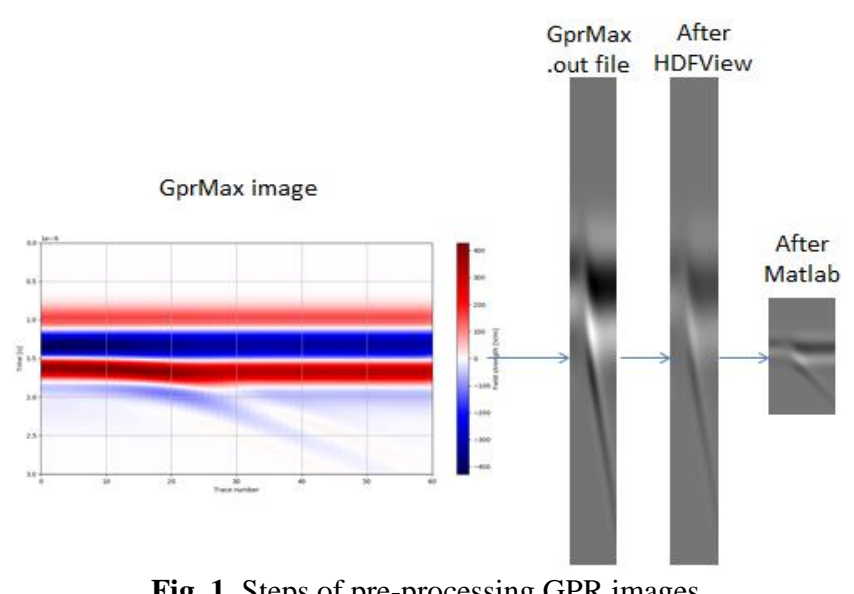

Fig. 1. Steps of pre-processing GPR images.

After rescaling all of the B-scans, they were changed from RGB to grey scale, and then resized from $637 \times 60$ to $112 \times 60$, in order to reduce the amount of memory needed to train the proposed CNNs. Hence, the pre-processing steps included rescaling, changing color format, and finally resizing, without a need of any complex pre-processing steps (e.g. edge detection, segmentation, and support vector machine (SVM) classifiers).

\subsection{System Architecture}

The proposed system architecture, as shown in Figure 2, takes a set of GPR images as input and applies certain operations on them according to each proposed CNN. The output of CNNs is a probability of this image being of a certain class (e.g. concrete, PVC, and metal materials).

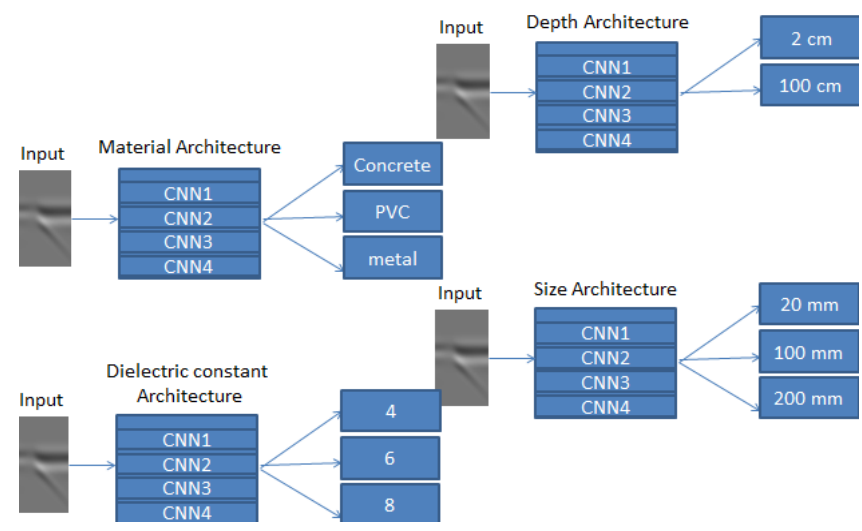

Fig. 2. Proposed system architecture. 
Four CNN models are used in this study. CNN1 and CNN2 are newly proposed and then compared with CNN3 and CNN4 which are suggested by [14] and [1], respectively. Each model is used to classify 2D cylinders' scans according to four classification categories: depth, material, size, and the dielectric constant of the burying medium. The proposed CNN models are illustrated in Figure 3. CNN1 includes 4 blocks, and its first and second blocks are exactly the same. Both blocks include a convolutional layer, a batch normalization layer, a ReLu layer, and a max pooling layer. The third block is similar to the previous two blocks in the layers and the order of these layers, but it excludes the max pooling layer. The last block contains a fully connected layer, a softmax layer, and an output layer. CNN2 includes 2 blocks, the first block contains a convolutional layer, a ReLu layer, and a max pooling layer. The second and final block contains a fully connected layer, a softmax layer, and an output layer. CNN3 and CNN4 include 5 and 3 blocks respectively. Table 1 shows the characteristics of these four CNNs in details.

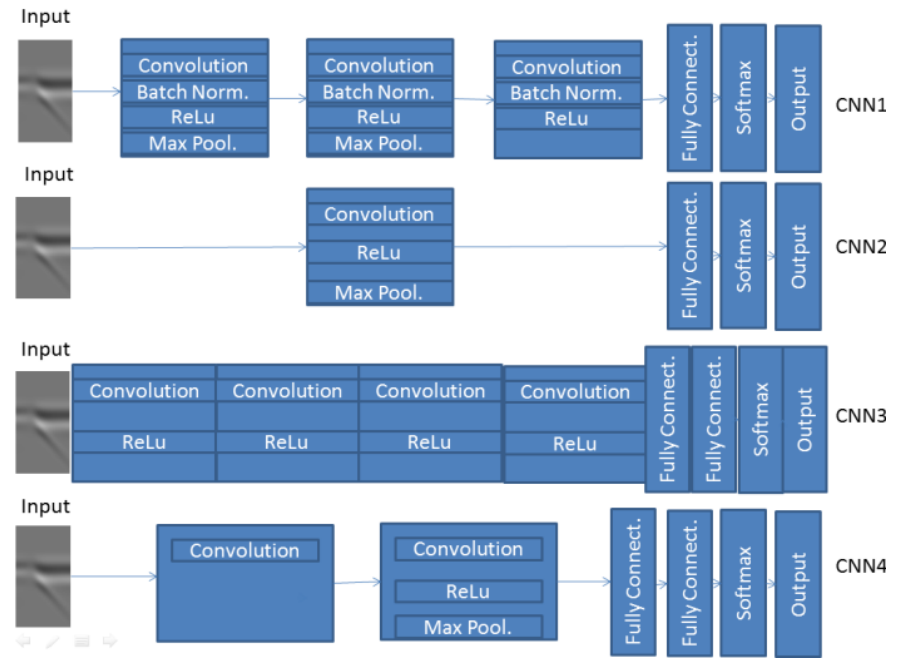

Fig. 3. Proposed CNN models.

Table 1. The characteristics of the used CNNs.

\begin{tabular}{lccccc}
\hline Proposed/Recommended & $\begin{array}{c}\text { Number of } \\
\text { blocks }\end{array}$ & $\begin{array}{c}\text { Number of } \\
\text { layers }\end{array}$ & $\begin{array}{c}\text { Filter } \\
\text { size }\end{array}$ & $\begin{array}{c}\text { Number of } \\
\text { filters }\end{array}$ \\
\hline CNN1 & Proposed & 4 & 15 & $3 * 3$ & 16,32, and 64 \\
CNN2 & Proposed & 2 & 7 & $5 * 5$ & 20 \\
CNN3 & Recommended by [14] & 5 & 15 & $3 * 3$ & $16,16,32,32$ \\
CNN4 & Recommended by [1] & 3 & 9 & $5 * 5$ & 20 \\
\hline
\end{tabular}

\section{Numerical Results}

The four convolutional models were trained using $75 \%$ of the synthetic data mentioned in Section 2.1 and tested using $25 \%$ of that data. Training and testing were conducted on a single CPU using stochastic gradient descent with momentum (SGDM).

The steps of the experiment are:

- Finding the effect of adjusting different training options on the accuracy of the four CNN models. The investigated options were:

$\circ \quad$ The initial learning rate $=0.0001$. 
- Learn rate drop factor $=50 \%$ and learn rate drop period $=4$ epochs, suggested by [14].

- The maximum number of epochs (as needed to get $90 \%$ accuracy).

O Other training options were set to default values.

- Training the four models using the best combination of the previously mentioned options, and then finding which architecture performs the best.

- Find which of the four classification categories works best.

Results show that there is no single training option that worked the same for the four suggested CNNs. Adjusting the initial learning rate and the learning rate drop parameters worked well with some models but poorly with others, while maximizing the number of epoch which always improved accuracy. A combination of adjusted initial learning rate, learning rate drop factor, learning rate drop period (Table 2), and maximum number of epochs (Table 3) improved the performance of the four CNN models in classifying the GPR dataset. The four models were able to achieve $90 \%$ classification accuracy, but with different number of epochs. The best models were the ones that needed less epochs to classify GPR images. This is because the less the epochs the less the classification time. Furthermore, as shown in Table 3, the proposed CNN1 showed the best performance using the suggested combination of training options in Table 2 with learning rate drop factor $=0.5$ and learning rate drop period $=4$ epochs. CNN1 was able to classify GPR images according to their dielectric constant, depth, material, and size with an average of 60.7 epochs. CNN2 needed an average of 69 epochs. CNN3 and CNN4 took an average of 151.7 epochs and 163.7 epochs, respectively.

CNN1 performed the best because it is differentiated from the other previously mentioned networks by having three convolutional layers with different amount of $3 * 3$ filters. Having a larger number of smaller filters improves the accuracy of architecture because they are able of catching more features [14]

In addition it was found in Table 3 that classifying B-scans based on cylinders' materials was more accurate than other classification properties with any of the four CNNs. Classifying GPR images based on cylinders' materials needed only 86.7 epochs on average while classifying on the depth, size, and dielectric constant need an average of 94.5, 98.7, and 101.5 epochs, respectively.

Table 2. The best combination of the adjusted training options for each proposed CNN.

\begin{tabular}{ccccc}
\hline Adjusted option & CNN1 & CNN2 & CNN3 & CNN4 \\
\hline Initial learning rate & & x & & x \\
Learning rate drop factor and learning rate drop period & x & x & x & \\
\hline
\end{tabular}

Table 3. The needed number of epochs for each model to achieve an accuracy of at least $90 \%$ using the training options combination in Table 2 .

\begin{tabular}{cccccc}
\hline Classification categories & CNN1 & CNN2 & CNN3 & CNN4 & Average \\
\hline Dielectric constant & $49 \mathrm{e}$ & $53 \mathrm{e}$ & $146 \mathrm{e}$ & $158 \mathrm{e}$ & $101.5 \mathrm{e}$ \\
Depth & $90 \mathrm{e}$ & $111 \mathrm{e}$ & $100 \mathrm{e}$ & $153 \mathrm{e}$ & $94.5 \mathrm{e}$ \\
Material & $56 \mathrm{e}$ & $60 \mathrm{e}$ & $136 \mathrm{e}$ & $200 \mathrm{e}$ & $86.7 \mathrm{e}$ \\
Size & $48 \mathrm{e}$ & $52 \mathrm{e}$ & $225 \mathrm{e}$ & $144 \mathrm{e}$ & 98.7 \\
Average & $60.7 \mathrm{e}$ & $69 \mathrm{e}$ & $151.7 \mathrm{e}$ & $163.7 \mathrm{e}$ & - \\
\hline
\end{tabular}




\section{Conclusion}

In this paper, cylinders' B-scans were classified according to their depth, size, material, and the dielectric constant of the underground medium using four different models of CNNs. Two of these networks were newly proposed in this study, and the other two were from [1] and [14]. These CNNs were trained using different adjusted training options including initial learning rate, learning rate drop factor, and learning rate drop period. These three parameters had impact on the performance of the used models. With the best combination of training options CNN1 showed superior performance due to the use of a deep network with a large number of small filters. It was found that the best performance was obtained when GPR images were classified according to the materials of buried cylinders.

\section{References}

[1] Lombardi, F.: Landmine detection from GPR data using convolutional neural networks. European Signal Processing Conference (EUSIPCO). (2017)

[2] Huang, Y.: Buried pipe detection by ground penetrating radar using the discrete wavelet transform. Elsevier. Vol. 37, pp. 440-448 (2010)

[3] Jaedicke, C.: Avalanche victim search by ground penetrating radar. The International Snow Science Workshop, Jackson Hole, Wyoming (2004)

[4] Fruehauf, F.: Experiments and algorithms to detect snow avalanche victims using airborne ground-penetrating radar. IEEE Transactions on Geoscience and Remote Sensing, Vol. 47, Issue: 7, pp. $2240-2251(2009)$

[5] Roberts R.: Ground penetrating radar for the detection of liquid contaminants. Elsevier. Vol. 33, pp. 195-207 (1995)

[6] Xiujun, G.: Detecting three types of contaminated soil with ground penetrating radar. International Conference on GPR. (2012)

[7] Candra, P.: 2-D entropy and short-time fourier transform to leverage GPR data analysis efficiency. IEEE Transactions on Instrumentation and Measurement, Vol. 64, Issue: 1 (2015)

[8] Hashim, M.: Material classification of underground utilities from gpr images using DCT-based SVM approach. IEEE Geoscience and Remote Sensing Letters, Vol. 10, (2013)

[9] Huston, D.: Underground object characterization based on neural networks for ground penetrating radar data. SPIE. (2016).

[10] Li, P.: GPR identification of voids inside concrete based on Support Vector Machine (SVM) algorithm. The 14th International Conference on Ground Penetrating Radar (GPR). (2012)

[11] Nash, R.: An introduction to convolutional neural networks. arXiv:1511.08458 (2016)

[12] Morton, K.: Recognizing subsurface target responses in ground penetrating radar data using convolutional neural networks. SPIE. (2015)

[13] Reichman, D. Improving convolutional neural networks for buried target detection in ground penetrating radar using transfer learning via pretraining. SPIE. (2017)

[14] Reichman, D.: Some good practices for applying convolutional neural networks to buried threat detection in ground penetrating radar. The 9th International Workshop on Advanced Ground Penetrating Radar. (2017) 